Technological University Dublin ARROW@TU Dublin

\title{
Electrohydrodynamic Printing of a Dielectric Elastomer Actuator and Its Application in Tunable Lenses
}

\author{
Liang Jiang \\ Qindao University \\ Yuhao Wang \\ Qingdao Unviersity \\ Xueqin Wang \\ Qingdao University
}

See next page for additional authors

Follow this and additional works at: https://arrow.tudublin.ie/aegart

\section{Recommended Citation}

Jiang, L. et al. (2021). Electrohydrodynamic printing of a dielectric elastomer actuator and its application in tunable lenses. Composites Part $A$,

This Article is brought to you for free and open access by the Applied Electrochemistry Group at ARROW@TU Dublin. It has been accepted for inclusion in Articles by an authorized administrator of ARROW@TU Dublin. For more information, please contact arrow.admin@tudublin.ie, aisling.coyne@tudublin.ie, gerard.connolly@tudublin.ie.

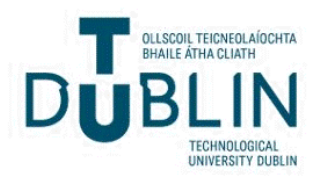




\section{Authors}

Liang Jiang, Yuhao Wang, Xueqin Wang, Fanggang Ning, Shipeng Wen, Yanfen Zhou, Shaojuan Chen, Tony Betts, Stephen Jerrams, and Fenglei Zhou 


\title{
Electrohydrodynamic printing of a dielectric elastomer actuator and its application in tunable lenses
}

Liang Jiang ${ }^{1,2}$, Yuhao Wang ${ }^{1}$, Xueqin Wang ${ }^{1}$, Fanggang Ning ${ }^{1}$, Shipeng Wen ${ }^{3}$, Yanfen Zhou $^{1,2, *}$, Shaojuan Chen ${ }^{1,2}$, Anthony Betts ${ }^{4}$, Stephen Jerrams ${ }^{4}$, Fenglei Zhou ${ }^{1,5}$

${ }^{1}$ College of Textiles and Clothing, Qingdao University, Qingdao 266071, China

${ }^{2}$ Eco-Textile Collaborative Innovation Center, Qingdao University, Qingdao, 266071, China

${ }^{3}$ Beijing Engineering Research Center of Advanced Elastomers, Beijing University of Chemical Technology, Beijing 100029, China

${ }^{4}$ Applied Electrochemistry Group, Technological University Dublin, City Campus, FOCAS, Kevin St, Dublin D08 NF82, Ireland

${ }^{5}$ Centre for Medical Image Computing, University College London, London, WC1V 6LJ, UK

*Corresponding author: Yanfen Zhou (yanfen.zhou@qdu.edu.cn)

\begin{abstract}
Optical lenses driven by dielectric elastomer (DE) actuators with tunable focal lengths are presented here. They are inspired by the architecture of the crystalline lens and the ciliary muscle of the human eye and have prompted a growing interest. The most commonly used DEs in tunable lenses have often required highly transparent films and also the need to encapsulate clear liquid silicone to act as the lens. There is a restriction on the properties of the tunable lens imposed by materials limitations. Here, the
\end{abstract}


fabrication of a fully 3D printed tunable lens with an inhomogeneous structure is described. It exhibited a 29\% change in focal length from $33.6 \mathrm{~mm}$ to $26.1 \mathrm{~mm}$ under a dynamic driving voltage signal control. Furthermore, it displayed excellent stability when the focal length was tuned from far to near (30.1 mm to $25.3 \mathrm{~mm}$ ) for 200 cycles. The tunable lens obtained mimics the working principle of the human eye in auto adjusting the focal length and has evident potential applications in imaging, information storage, beam steering and bifocal technology.

\section{Introduction}

One of the most significant components in lifelike robots is the embedded soft actuator which provides the sensitive movements that humans are capable of [1]. For example, eyes in soft robots should be able to mimic the ability of human eyes to tune the focal length fluently, precisely and with a quick response [2]. The lens in the eye is suspended in place by its suspensory ligament, a ring of fibrous tissue attached to the lens at its equator and connected to the ciliary body. Tuning the focal length of the lens is realized by the contraction and relaxation of the ciliary muscle, as illustrated in Figure 1(a) and (b). However, the eyes in the majority of current robots are based on digital cameras which tune focus by mechanically moving rigid components with the limitations of bulk and inertia, consuming high energy. In order to overcome these drawbacks, many soft actuation based-approaches such as hydraulic/pneumatic pressure [3], electromagnetic waves [4], electrochemistry [5], electro wetting [6], dielectrophoretic forces [7] and stimuli-responses [8], have been proposed to control the curvature of liquid droplet with the ability to tune the focal length. However, these soft actuators are 
subject to serious limitations such as having slow response speeds and short lifetimes, or alternatively they must be tethered to external bulky controllers that cannot be integrated [9-11]. Liquid-encapsulating technology which can utilise hemispherical surfaces of liquid with thin polymeric materials or filling liquid between a pair of dielectric elastomer (DE) films opens a promising route due to offering quick responses and light weights [2, 11]. This concept was initially proposed by Carpi et al. [8], in whose study a low-mechanical loss silicone was used as the dielectric membrane. It has been regarded as the world's fastest tunable lens with a long shelf life. Since then, extensive efforts have been made in the development of dielectric elastomer actuators (DEA) driven tunable lenses possessing superior electromechanical properties.

(a)

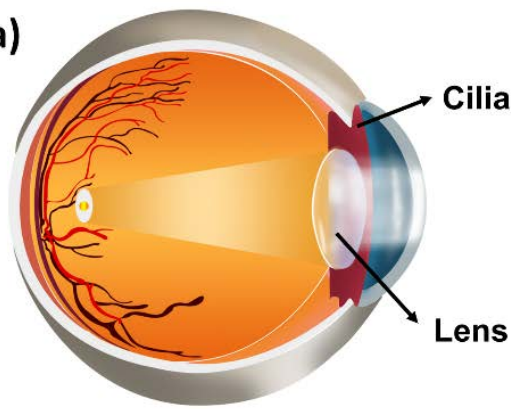

(c)

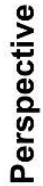

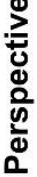

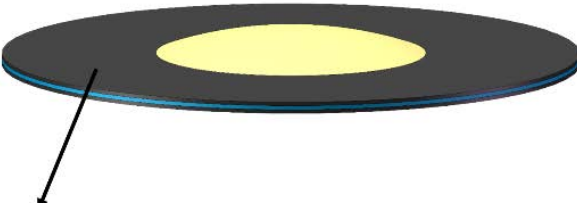

(b)

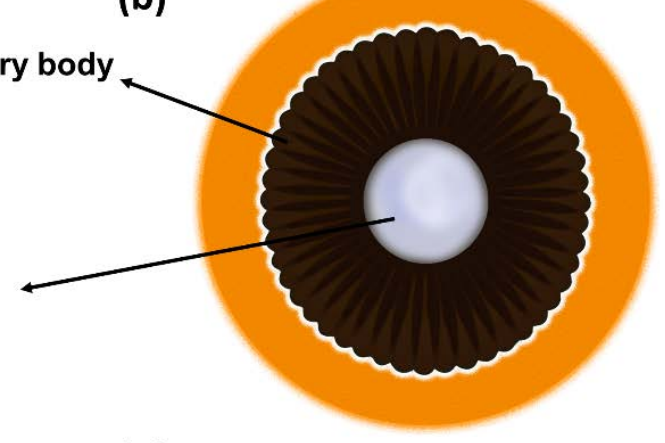

(d)

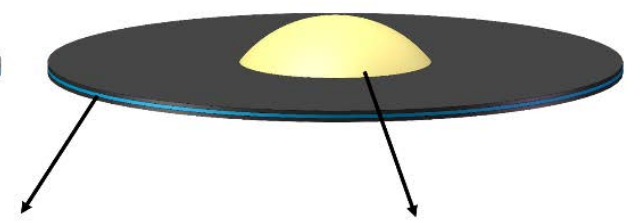

Dielectric elastomer

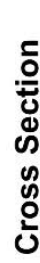

Compliant electrode

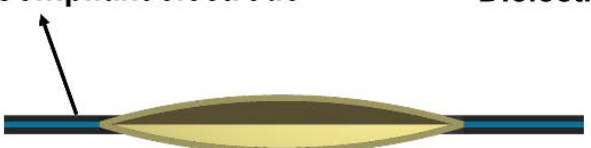

Voltage Off

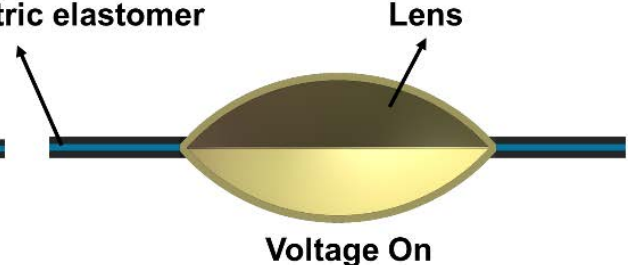


(a)

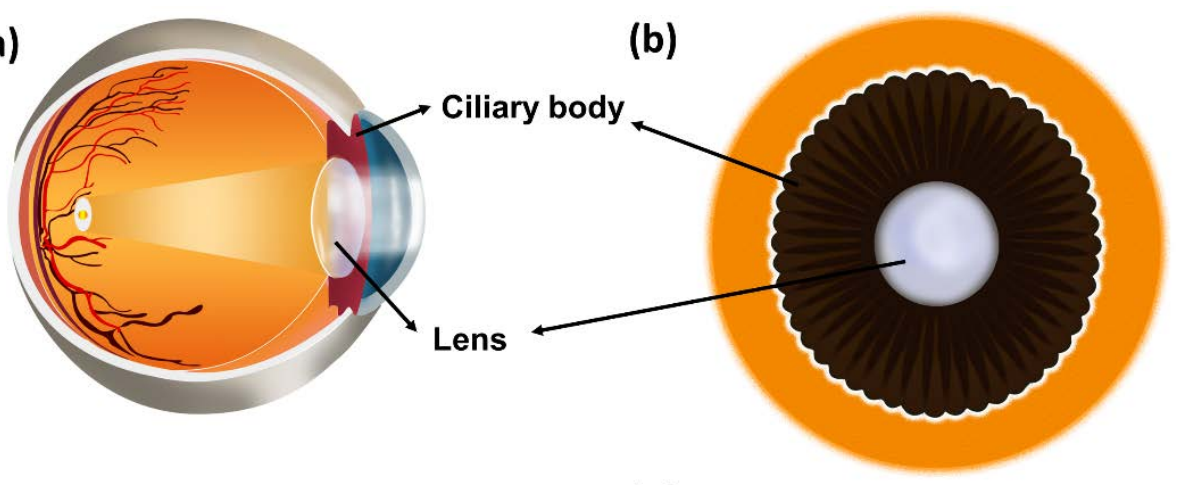

(c)
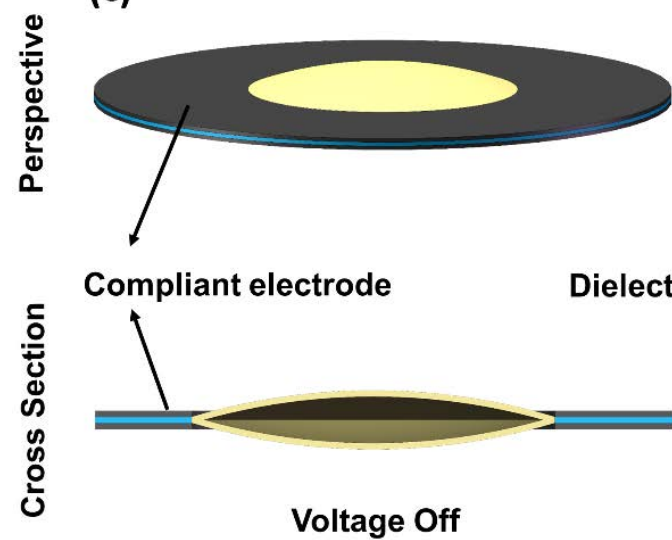

(d)
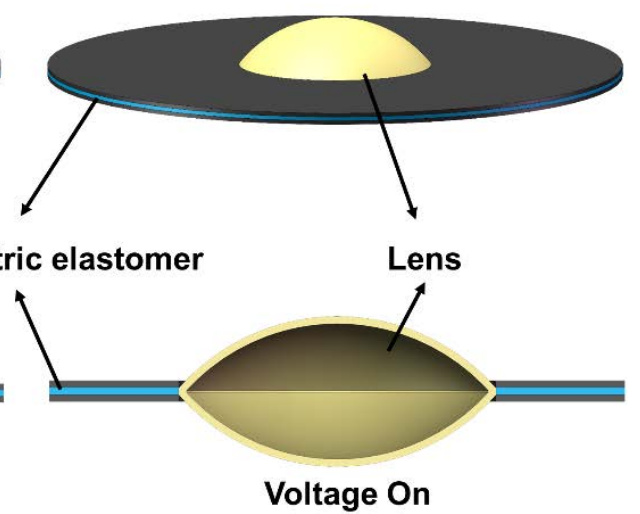

Figure 1 (a) a diagram of the human eye and (b) a front view of the eye; the perspective and cross sectional schematic of a dielectric elastomer based tunable lens.

(c) With the voltage off the focal length is distanced. (d) With the voltage on the focal length becomes shorter.

DEs $[12,13]$ are electrostrictive polymers and can achieve large actuated strain (> 380\%), quick response speeds and high electromechanical energy densities ( 3.4 $\mathrm{MJ} / \mathrm{m}^{3}$ ) [14-18], together with light weight, low cost and absence of noise pollution [9, 19-24]. The working mechanism behind a tunable lens using a DE actuator (DEA), which is a DE ring film coated with a compliant electrode assembled into a tunable lens, is illustrated in Figure 1(c) and (d). Prior to its use, the tunable lens is pre-stretched and clamped by a frame. When a voltage is applied, the DEA is expanded by the creation of a Maxwell stress which can be determined by Equation (1). Then if the lens is 
squeezed, with its diameter becoming smaller and its height becoming larger, it exhibits a short focal length; When the voltage is off, the DEA recovers its shape and the lens retains its original dimensions, leading to a greater focal length.

$$
\sigma_{V}=\varepsilon^{\prime} \varepsilon_{0}(\Phi / h)^{2}=\varepsilon^{\prime} \varepsilon_{0} \varphi^{2}
$$

where $h$ is the thickness of the DE, $\varepsilon^{\prime}$ is the dielectric constant of the DE material, $\varepsilon_{0}$ is the permittivity of free space $\left(8.85 \times 10^{-12} \mathrm{~F} / \mathrm{m}\right), \varphi$ is the electric field strength which is equal to the applied high voltage $(\Phi)$ divided by the thickness of the DE $(h)$.

However, there are only a few transparent DE materials reported, including pure silicone and VHB 4910, that can be used for creating high performance DE based lenses. It is well known that the electromechanical properties of DEs could be enhanced by blending with other polymers [25] or incorporating dielectric fillers [26], but making these changes could result in the transparency of the DEs becoming low. Therefore, it remains a challenge to create $\mathrm{DE}$ actuated tunable lenses with a high dielectric constant together with both transparency and high electromechanical properties. A potential approach is to design a hybrid structure comprising a transparent and stretchable elastomeric sphere as the lens and a transition ring of DE composite as the actuator. A major step towards realizing this structure is to bind the two components together without compromising the structures evenness and avoiding the formation of defects. Inkjet printing is an additive manufacturing technique offering the advantages of high manufacturing speeds, no requirement of moulds and suitability for programmably creating complex and intricate structures [27]. Inkjet printing has been employed to fabricate DEs and their corresponding compliant electrode [28]. However, this method 
can only print plane figures and the quality of the printed structure is restricted by the material parameters such as viscosity and surface tension. Therefore, a highly efficient, simple and low-cost fabrication technique for structuring DEs is highly desirable. Electrohydrodynamic (EHD) printing, which is an attractive high resolution (down to $100 \mathrm{~nm}$ ) direct writing technique uses an electric field rather than a thermal or acoustic field to create the fluid flows necessary for delivering inks containing high content fillers to a substrate [27, 29]. Therefore, it can be utilized for fabricating polymer composites with various shapes and dimensions. In this research, a hybrid structured tunable lens driven by a DEA was generated using EHD printing. A silicone rubber (SR) based ink was adopted to pattern the encapsulating layer of the lens with filled transparent silicone liquid and a copper phthalocyanine (CuPc) filled SR ink which is highly dielectric for fabricating the DEs driven component. The actuation performance and the working stability of DE driven tunable lenses were also investigated.

\section{Experimental}

\section{Materials}

Two-component silicone LSR 4305 was provided by Bluestar Ltd., USA. Copper phthalocyanine (CuPc) and n-heptane were purchased from Sinopharm Chemical Reagent Co., Ltd. (Shanghai, China). The lens-filling fluid was a silicone pre-polymer (Sylgard 184) with a refractive index of 1.43 purchased from Down Corning, USA. The commercially available conductive carbon grease NYOGEL 756 G, which was used as the compliant electrode in this work, was supplied by Nye Lubricants, Inc., Fairhaven, MA, USA. 


\section{EHD printing of DE driven tunable lenses}

DE membranes used to construct tunable lenses were printed by using a custom-built EHD 3D printing system as shown schematically in Figure 2. The EHD printing system consists of a high voltage power supply (HPSN 1220, LANYI, Zhejiang, China), an ink supplier, an ultrasonic device, a digital camera (WP-UT500, Shenzhen, China) and a conductive metal substrate which is made of 304 austenitic stainless steel fixed onto a programmable three-axis translation stage moving at a speed of $8 \mathrm{~mm} / \mathrm{s}$. The high voltage supply was connected to a nozzle with a blunt end (inner diameter of $210 \mu \mathrm{m}$, the outer diameter being $400 \mu \mathrm{m}$ ) and an electrically-grounded conductive substrate. The working distance between the blunt end and the conductive substrate is $2 \mathrm{~mm}$. The ink in the nozzle was supplied by a syringe pump (Longer Precision Pump Co., Ltd., Baoding, Hebei, China) with a typical flow rate of $0.05-0.07 \mu \mathrm{L} \cdot \mathrm{min}^{-1}$. During the printing process, the syringe was immersed in a tank filled with water allowing ultrasonication. A good dispersion of the CuPc particles in the ink was ensured by maintaining ultrasonication during printing. EHD 3D printing was initiated by gradually increasing the applied voltage, until firstly the pendant ink drop deformed into a conical shape and then elongated and fell onto the printing substrate. The morphology of the cone created in EHD 3D printing was recorded by the camera.

The tunable lens was comprised of a DE ring film as the driven part and the silicone liquid encapsulated in a transparent SR film serving as the lens. The fabrication process of a DE driven tunable lens is also illustrated in Figure 2. CuPc particles were firstly dispersed into n-heptane and ultra-sonicated for 5 min (Figure 2(a)). Then the mixture 
was added to the two-component SR solution and magnetically stirred for $10 \mathrm{~min}$ in order to obtain a good even dispersion. The pure SR ink was used to print transparent films (Figure 2(b)) and the SR with 3wt\% CuPc composite ink (denoted as SR/ 3wt\% CuPc ink) obtained was used to print a DE ring film with a diameter of $60 \mathrm{~mm}$ employing the EHD 3D printing system (Figure 2(c)). The printed film was left in an oven at $120^{\circ} \mathrm{C}$ for $1 \mathrm{~h}$ until the film was fully cured. Subsequently, the film obtained was placed on a disk with a circular hole having a diameter of $5 \mathrm{~mm}$ and placed under a vacuum to form a hemispherical cavity. The cavity was filled with $50 \mu \mathrm{L}$ silicone (Sylgrad 184, USA) (Figure 2(d)) and covered by another layer of transparent SR film in disk form which was fabricated by printing it separately (Figure 2(e)). Afterwards, a ring-shaped frame with $60 \mathrm{~mm}$ external diameter was printed along the edge of the disk SR film by using SR/3wt\% CuPc ink and then cured by heating at a temperature of $120^{\circ} \mathrm{C}$ (Figure 2(f)). By this process, finally, the DE driven tunable lens, having a thickness of $600 \mu \mathrm{m}$, was obtained. 
(a)

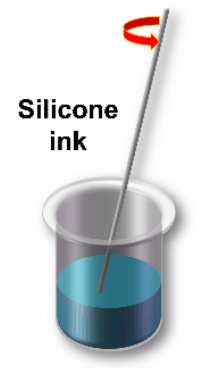

Ink preparation (b)

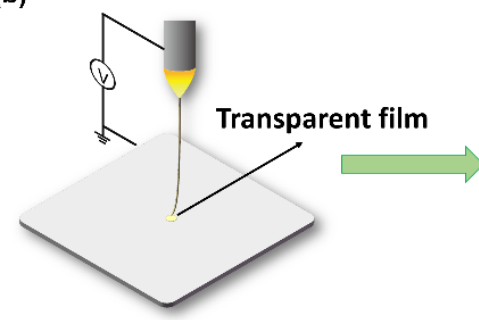

Transparent film printing (c)

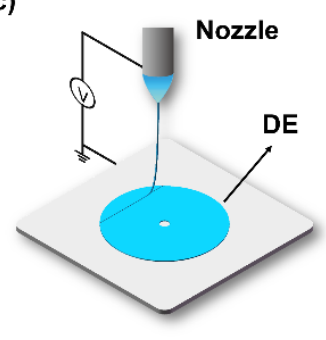

Bottom DE printing (e)

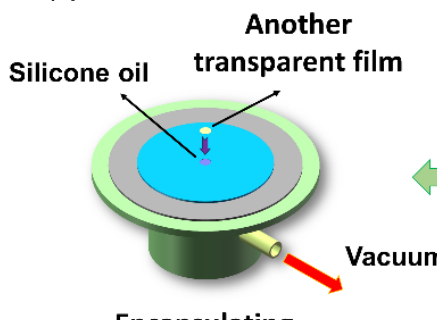

Encapsulating (d)

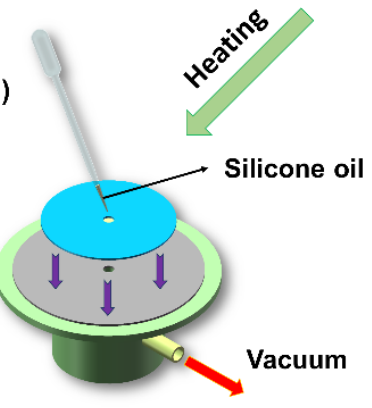

Silicone oil loading

(f)

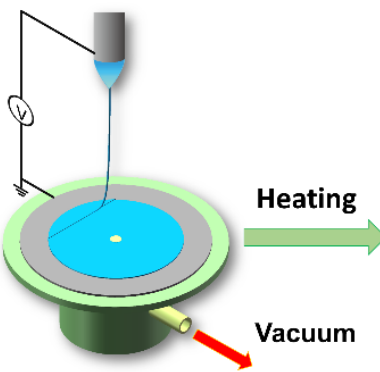

Top DE printing

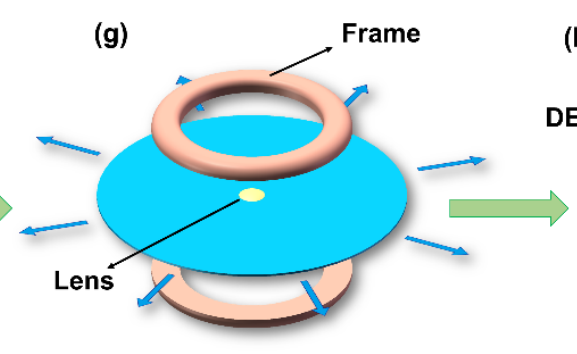

Pre-stretch (h) Complaint electrode

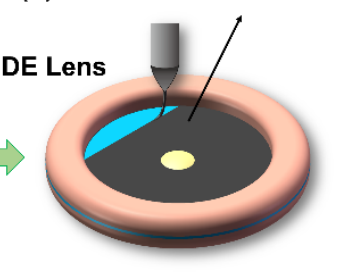

Electrode printing

Figure 2 Schematic illustration of the fabrication procedure of a DE driven tunable lens by using EHD printing.

The compliant electrode ink was prepared by filling 40 mL n-heptane in 10 g NYOGEL 756 G. Before patterning the electrode on the DE ring, the lens was pre-stretched to a diametral ratio of 1.8 by using a rig specifically designed for pre-stretching. Two annular frames were applied as the support to fix the pre-stretched lens (Figure 2(g)). Then, the compliant electrode was printed on the pre-stretched lens by using a plastic nozzle with voltage turned off along the programmed trajectory (Figure 2(h), Video S1). 
The DE film with the rig was reversed and the printing procedure repeated. The lens obtained with patterned electrodes was used for the electromechanical test.

\section{Characterization}

The 3D surface morphology of DE lens was observed using an ultra-depth 3D digital microscope (DVM6 M, Leica Microsystems Ltd., Germany).

The diameter of the tunable lens with the compliant electrode at selected voltages was measured on an electromechanical testing system mainly consisting of a computer, a high voltage power supply and a camera. A test rig was used to circumferentially clamp samples. The DE driven tunable lens was initially fixed onto the circular frames with a pre-stretch ratio of 1.8. The camera recorded the changes in diameter and area when an applied electric field was incrementally increased in voltage steps of $200 \mathrm{~V}$ at $3 \mathrm{~s}$ intervals until electric breakdown of the lens occurred. The initial area $\left(A_{0}\right)$ and the actuated area $\left(A_{1}\right)$ were measured automatically in real time by using LabVIEW. The area strain $\left(s_{a}\right)$ was calculated using Equation (2).

$s_{a}=\left|\left(A_{1}-A_{0}\right) / A_{0}\right|$

The change of focal length was measured with a specially designed setup containing a laser transmitter, a high voltage power supply, a camera, a pre-stretch rig and a container with soybean milk which was applied as the colloid. The laser beam travelled horizontally towards the lens edge and converged to pass the focus point when it went out from the lens. When the laser beam passed through the colloid, owing to Tyndall effect, the laser beam path was clearly presented in the colloid. The angle change of the laser beam path was recorded by the camera. The intersection point of the lens 
centerline and the laser beam line was the focus point of the lens. Consequently, the focal length was calculated as described in the next section.

\section{Results and discussion}

In the EHD printing process, the charged liquid droplet at the tip of the nozzle was gradually deformed due to the Coulombic force in an electric field formed between the nozzle and the grounded substrate when the voltage was applied, resulting in the droplet changing from a hemispherical shape into a conical shape. When the applied voltage reached a threshold value, a critical conical shape called a Taylor cone was formed, from which a jet was initiated by a further increase in applied voltage (as illustrated in Figure 3(a) and (b)). The force acting on the liquid cone is presented in Figure 3(b). It has been reported that the cone-jet mode was formed only when the electrostatic force $\left(F_{e s}\right)$ was larger than sum of the force due to viscosity $\left(F_{\mu}\right)$ and surface tension $\left(F_{s t}\right)$ [30], as shown in Equation (3)

$F_{e s}>F_{\mu}+F_{s t}$

The viscosity and surface tension of inks can be influenced by the concentration of solvent, the relationship was presented in Figure 3(c). The SR/3wt\% CuPc ink with a solute concentration of $20 \%$ had a viscosity of $6.98 \pm 0.58 \mathrm{mPa} \cdot \mathrm{s}$ and a surface tension of $18.43 \pm 0.49 \mathrm{mN} / \mathrm{m}$. It can be seen that the viscosity increased with increasing solvent content in the SR/3wt\% CuPc ink, while the surface tension decreased with the increase in ink concentration. This is because the n-heptane had a lower viscosity and higher surface tension than that of the pure silicone. Furthermore, the cone angle and jet diameter, which can be affected by printing voltage, ink supply rate and concentration 
of ink, are important criteria for evaluating a cone-jet mode during EHD printing. The influence of these factors on the cone angle and the jet diameter was depicted in Figure 3(d-i).

(a)
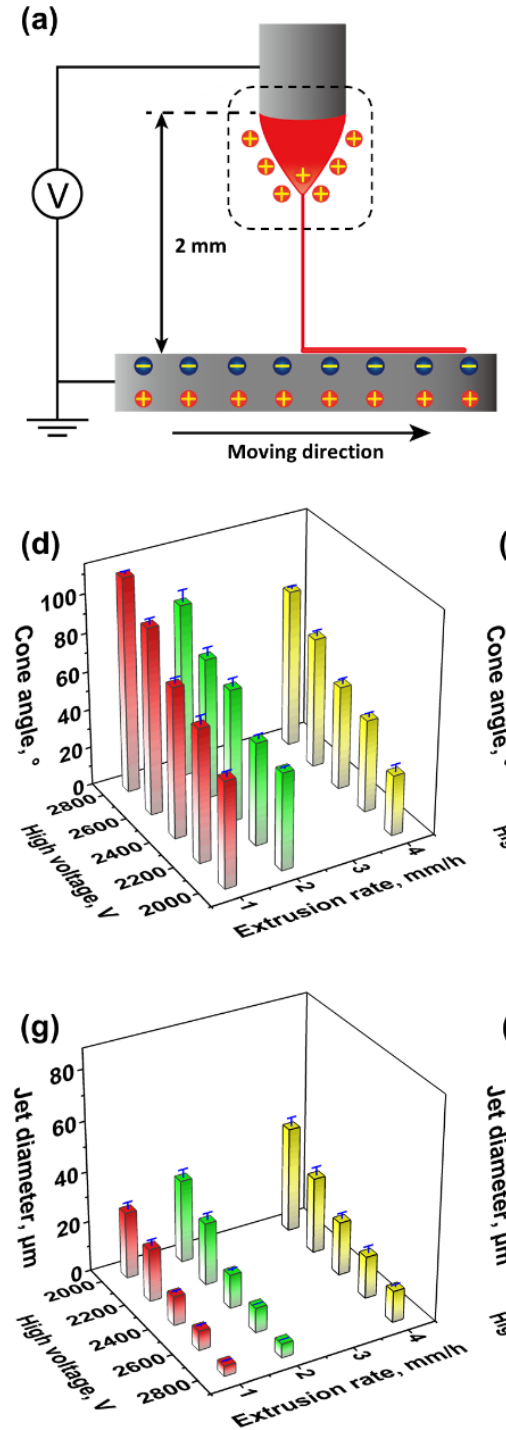

(b)
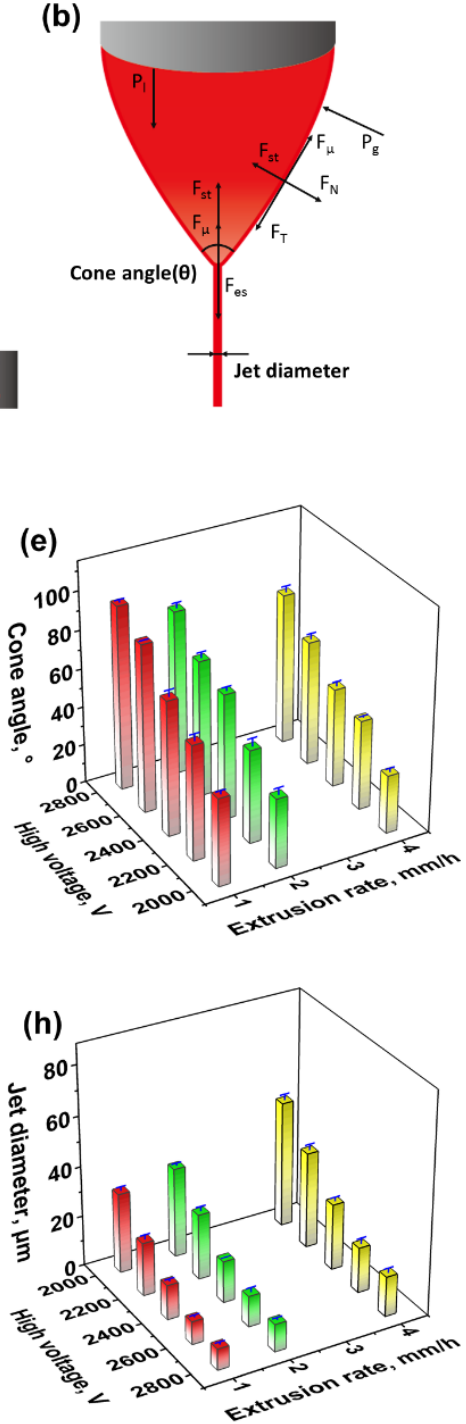

(c)
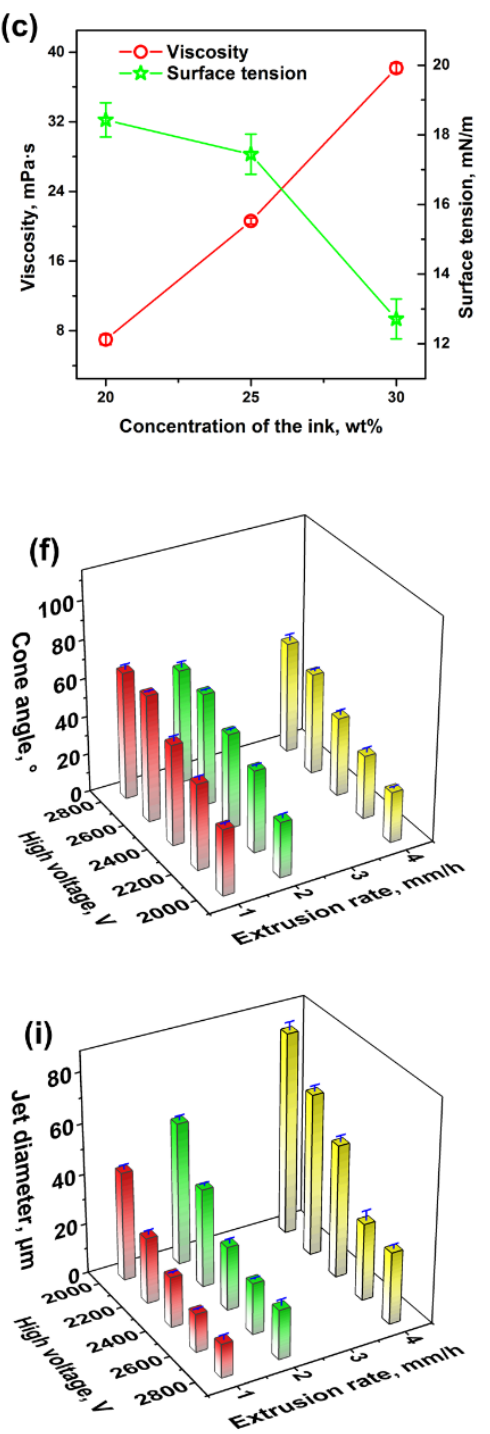

Figure 3 (a) Schematic of EHD. (b) Force acting on the liquid cone. (c) The dependence of viscosity and surface tension on the concentration of the composite ink; Histogram of cone angle related to high voltage and extrusion rate with an ink concentration of (d) $20 \mathrm{wt} \%$, (e) $25 \mathrm{wt} \%$ and (f) $30 \mathrm{wt} \%$. Histogram of the jet diameter related to high voltage and extrusion rate with an ink concentration of (g) 20 
wt\%, (h) $25 \mathrm{wt} \%$ and (i) $30 \mathrm{wt} \%$.

It was found from Figure 3(d-i) that the cone angle was increased and the jet diameter was decreased as the voltage rose from $2000 \mathrm{~V}$ to $2800 \mathrm{~V}$ for SR/3wt\% CuPc inks with solute concentrations of $20 \mathrm{wt} \%$ and $25 \mathrm{wt} \%$. This is because for a constant ink concentration and a constant extrusion rate, the increased high voltage induced a higher electrostatic force $\left(F_{e s}\right)$ and consequently led to a faster jet flow speed, then a shorter cone formed and the cone angle became larger [31]. Meanwhile, a larger tangential electrostatic force generated by the increased voltage induced a stronger backflow at the center of the cone, resulting in a decrease in jet diameter [32, 33]. For the ink with a solute concentration of $30 \mathrm{wt} \%$, the jet diameter decreased with the increasing applied voltage, while the cone angle increased with increasing voltage till $2600 \mathrm{~V}$ and then became larger when the voltage was increased further to $2800 \mathrm{~V}$ where the unstable printing occurred with a whip jet (seen from Figure 3(i)). Figure 3(d-i) also showed that the cone angle was decreased with the increased ink extrusion rate, while the jet diameter was increased when the ink extrusion rate was increased. This is because the increased ink supply rate directly led to an extension of the cone in order to survive in the high electric field, resulting in a decrease in the cone angle. Besides, owing to the increased ink supply rate, it is hard for the relatively weak backflow to reverse the ink inside the cone, allowing a thicker jet to be ejected [32]. Furthermore, it can be observed from Figure 3(d-i) that a smaller cone angle and a thicker jet were formed when the concentration of ink was increased from $20 \mathrm{wt} \%$ to $30 \mathrm{wt} \%$. The reason for this is probably that the increased content of silicone rubber in the ink resulted in an increased 
viscosity of ink, which required a higher electric field force to form a stable cone-jet mode. When the electric field force was insufficient, the cone was elongated and the cone angle decreased. Besides, ink with higher viscosity aided the jet formation, but it also thickened the jet at the same time [34, 35].

The above results showed that a smaller and more stable cone angle and jet diameter formed at a supply rate of $0.8 \mathrm{~mL} \cdot \mathrm{h}^{-1}$ and a printing voltage of $2600 \mathrm{~V}$ for all inks prepared in this work. For the purpose of improving the dielectric constant of printed DE films, the SR/3wt\% CuPc ink with a solute concentration of $30 \mathrm{wt} \%$ was employed for patterning the DE driven component of the tunable lens.

(a)

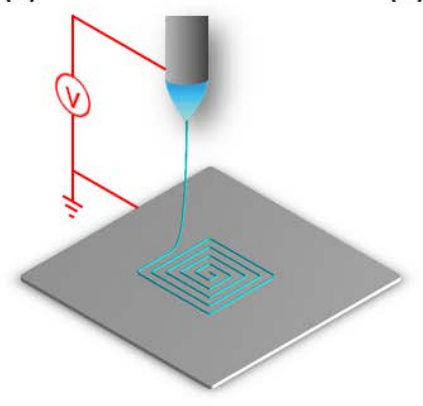

(c)

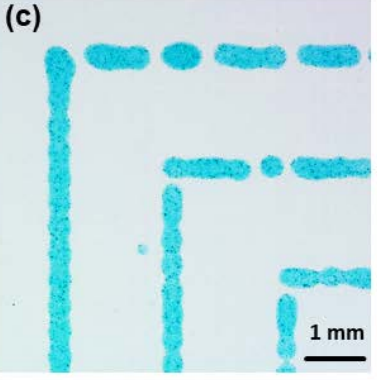

(f)

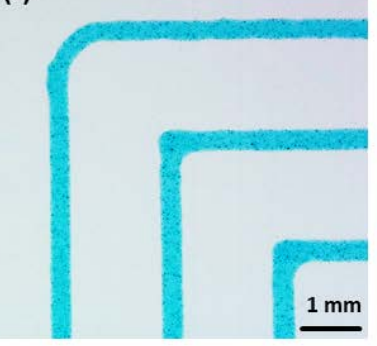

(b)

(d)

(g)
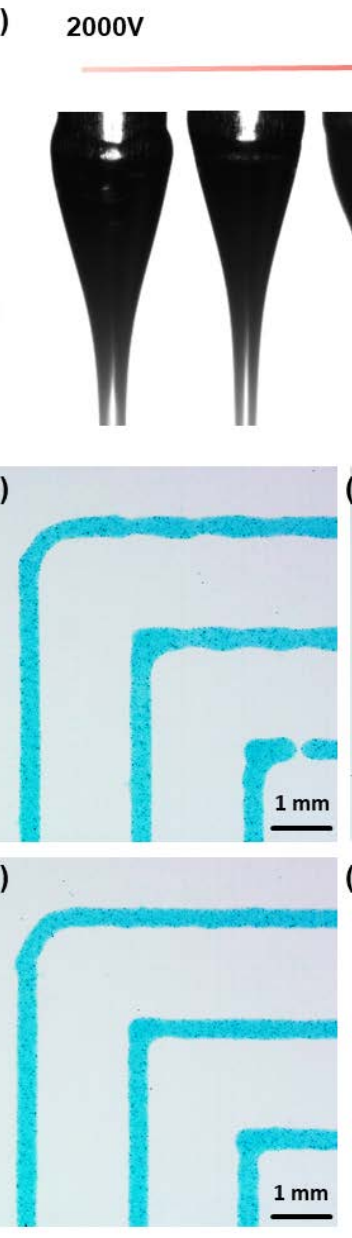

(e)

2800V
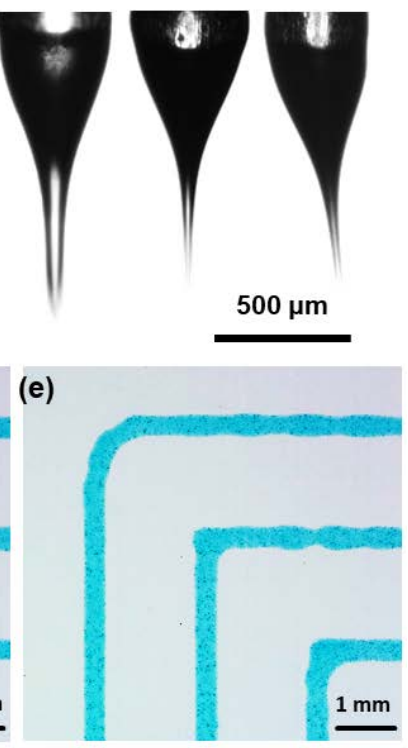

(h)

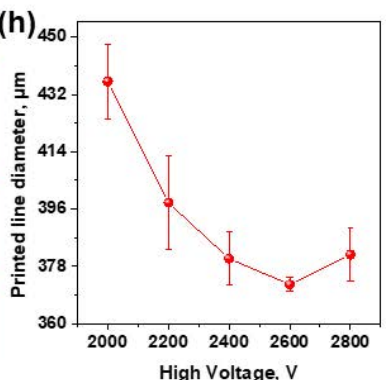

Figure 4 (a) the illustration of EHD printing for patterning lines on the conductive substate. (b) Jet behavior of the ink under the determined printing conditions at 
voltages from $2000 \mathrm{~V}$ to $2800 \mathrm{~V}$. The morphology of patterned lines for SR/3wt\% CuPc under different voltages: (c) 2000 V; (d) 2200 V; (e) 2400 V; (f) 2600 V and (g) $2800 \mathrm{~V}$. The plot showing the relation between the patterned line width and the applied voltage (h).

In order to investigate the printing resolution of the ink, the printing line was patterned as illustrated in Figure 4(a). The jet behavior of the ink under the determined printing conditions at voltages from $2000 \mathrm{~V}$ to $2800 \mathrm{~V}$ was shown in Figure 4(b). The cone angle and jet diameter were stated previously in Figure 3(f) and (i), respectively. Figures 4(cg) exhibited the morphology of printed lines of SR/3wt\% CuPc under the selected voltages. We can clearly see that the printed lines are clearly observed to have an even distribution with sharp corners indicating a high printing resolution. At the voltage of $2000 \mathrm{~V}$, the printed lines were intermittent with a more uneven width of about $436 \pm 12$ $\mu \mathrm{m}$. However, the breaks are barely visible in the printed lines when the applied voltage was above $2200 \mathrm{~V}$. This is probably because a shorter jet length was induced by a smaller electrostatic force at the lower voltage. It can be seen from Figure 4(f) that the width of the printed line decreased with increasing applied voltage below $2600 \mathrm{~V}$. The line printed at $2600 \mathrm{~V}$ was the most even and smooth with the smallest width of about $372 \pm 2 \mu \mathrm{m}$. The printed line width at $2800 \mathrm{~V}$ was about $382 \pm 8 \mu \mathrm{m}$, slightly larger than that of the printed line at $2600 \mathrm{~V}$. This was primarily due to the smearing of ink on collision with the substrate tending to widen the track. The jet tends to be thinner with increasing voltage, but at the same time the jet flow rate increases and obtains more kinetic energy leading to greater smearing. In addition, the unstable cone-jet occurring 
at $2800 \mathrm{~V}$ also contributed to the increase in line width. In this work, it was found that the adhesion and surface tension as the determinant factors during printing are similar for both pure SR and SR/3wt\% CuPc. Therefore, during printing the encapsulating layer of the lens, the same printing parameters as those of SR/3wt\% CuPc were adopted.

(a)

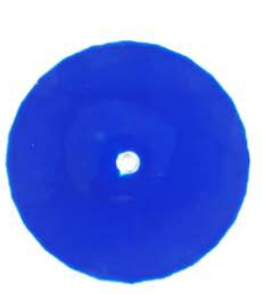

(d)

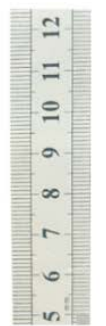

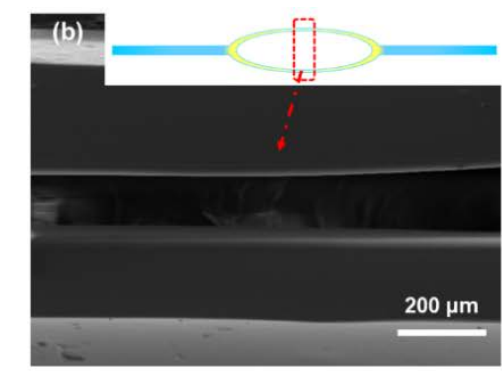

(e)

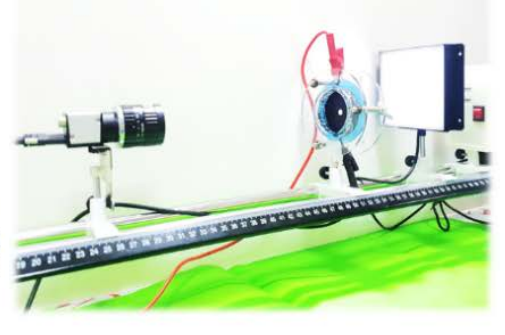

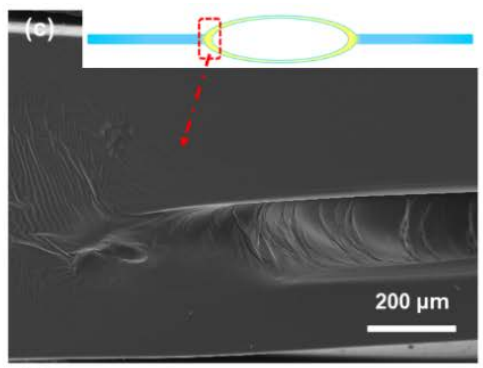

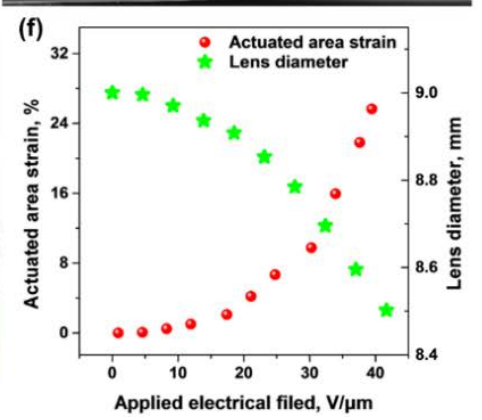

Figure 5 (a) Photograph of a printed lens. (b) The cross-section morphology of the lens at the centre (c) and at the lens edge. (d) Three-dimensional surface morphology of the printed lens observed by using an ultra-depth three-dimensional digital microscope. (e) Photograph of electromechanical test system for DEs. (f) The actuated area strain of SR/3wt\% CuPc and lens diameter against the applied electric field. A prototype of a DE driven tunable lens was then prepared by using an EHD printing process under an applied voltage of $2600 \mathrm{~V}$ (see Figure 5(a)). It can be seen from the figure that the DE ring film combined well with the centre lens. Figure 5(b) and (c) show the cross-section morphology of the centre lens and the interface between the centre lens and DE ring film, respectively. It can be seen that that the thickness of the SR film used for encapsulating the silicone oil was around $200 \mu \mathrm{m}$ and the thickness of 
the interface was about $700 \mu \mathrm{m}$. The edge between the centre lens and the DE ring film is hardly distinguished, indicating a good combination. The three-dimensional surface morphology of the printed lens was observed by using an ultra-depth three-dimensional digital microscope. As shown in Figure 5(d), the surface of the DE driven tunable lens was a uniform dome shape possessing a height of $3.2 \mathrm{~mm}$. The changes in the actuated area strain of the DE and the focal length of the lens relative to the increasing applied electric field were investigated by employing the setup presented in Figure 5(e). The results shown in Figure 5(f) revealed that the actuated area strain initially increased gradually with applied electric field and then drastically increased. The experimental procedure for obtaining the field-induced strain is the same as reported in the previous work [36]. This is mainly because the Maxwell stress causing the compression of the DEs has a second order relationship with the electric field. The SR/3wt\% CuPc had a large actuated area strain of up to $30 \mathrm{wt} \%$ at an applied electric field of $39 \mathrm{~V} / \mu \mathrm{m}$. With the applied electric field increasing from $0 \mathrm{~V} / \mu \mathrm{m}$ to $41 \mathrm{~V} / \mu \mathrm{m}$, the diameter of $\mathrm{DE}$ driven tunable lens was decreased from $9.0 \mathrm{~mm}$ to $8.5 \mathrm{~mm}$. 

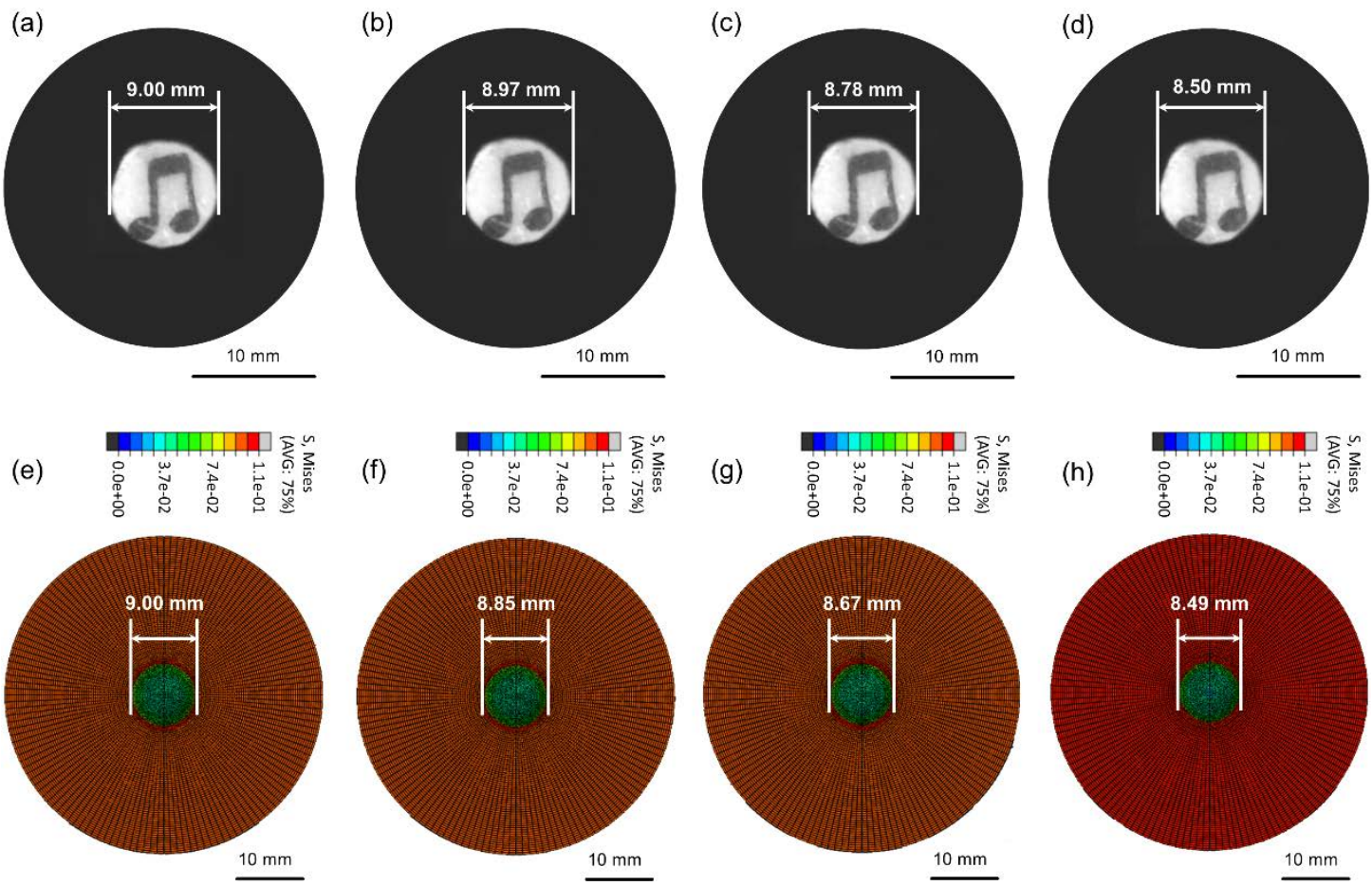

Figure 6 The deformation of a tunable lens under an applied electrical field of (a) 0

$$
\mathrm{V} / \mu \mathrm{m} \text {, (b) } 14 \mathrm{~V} / \mu \mathrm{m} \text {, (c) } 28 \mathrm{~V} / \mu \mathrm{m} \text { and (d) } 41 \mathrm{~V} / \mu \mathrm{m} \text {. (e-h) ABAQUS simulation of }
$$

deformation of the corresponding tunable lens in (a-d);

Figure 6(a-d) showed the deformation of a tunable lens under an applied electrical field ranging from 0 to $41 \mathrm{~V} / \mu \mathrm{m}$, whose process was shown in Video S2. In order to verify the repeatability of the experimental tunable lens deformation with electric field force, a numerical simulation of tunable lens deformation under the same operating conditions was performed by employing ABAQUS software. As tested in the previous work, the tensile strength of SR/3wt\% CuPc is $4.16 \pm 0.91 \mathrm{MPa}$ and the dielectric constant at the frequency of $10 \mathrm{~Hz}$ is 5.52 [36]. To model the electromechanical behavior of the lens, a two-step approach was taken. In the first step, equi-biaxial pre-stretch at a ratio of 1.8 was applied to the lens. In the second step, the Maxwell pressure as shown in Equation (1) was applied to the pre-stretched lens. The predicted results shown in Figure 6(e-h) 
illustrate that the lens diameter reduced from $9 \mathrm{~mm}$ to $8.1 \mathrm{~mm}$ under an applied electrical field rising from $0 \mathrm{~V} / \mu \mathrm{m}$ to $41 \mathrm{~V} / \mu \mathrm{m}$. The influence of applied electrical field onto the deformability of DEs was shown clearly in Video S3. It was found that the simulated lens deformation was slightly greater than the deformation achieved in experimentation. The uneven patterned electrode layer may have prevented the DE film achieving the same large voltage induced deformation, but it must be appreciated that the FE model is a mathematical model of the physical tests and discretization, boundary conditions and load applications are merely assumptions which will cause deviation from the physical test. Similarly, it is assumed that, during the simulation process, the DE composite film and the transparent SR film were perfectly bonded, while in the experimental preparation process, there were inevitable existing defects in the DE composite and in the overlapped region when mating two different parts. However, it is clear that the experimental results were in good agreement with the theoretical values. 
(a)
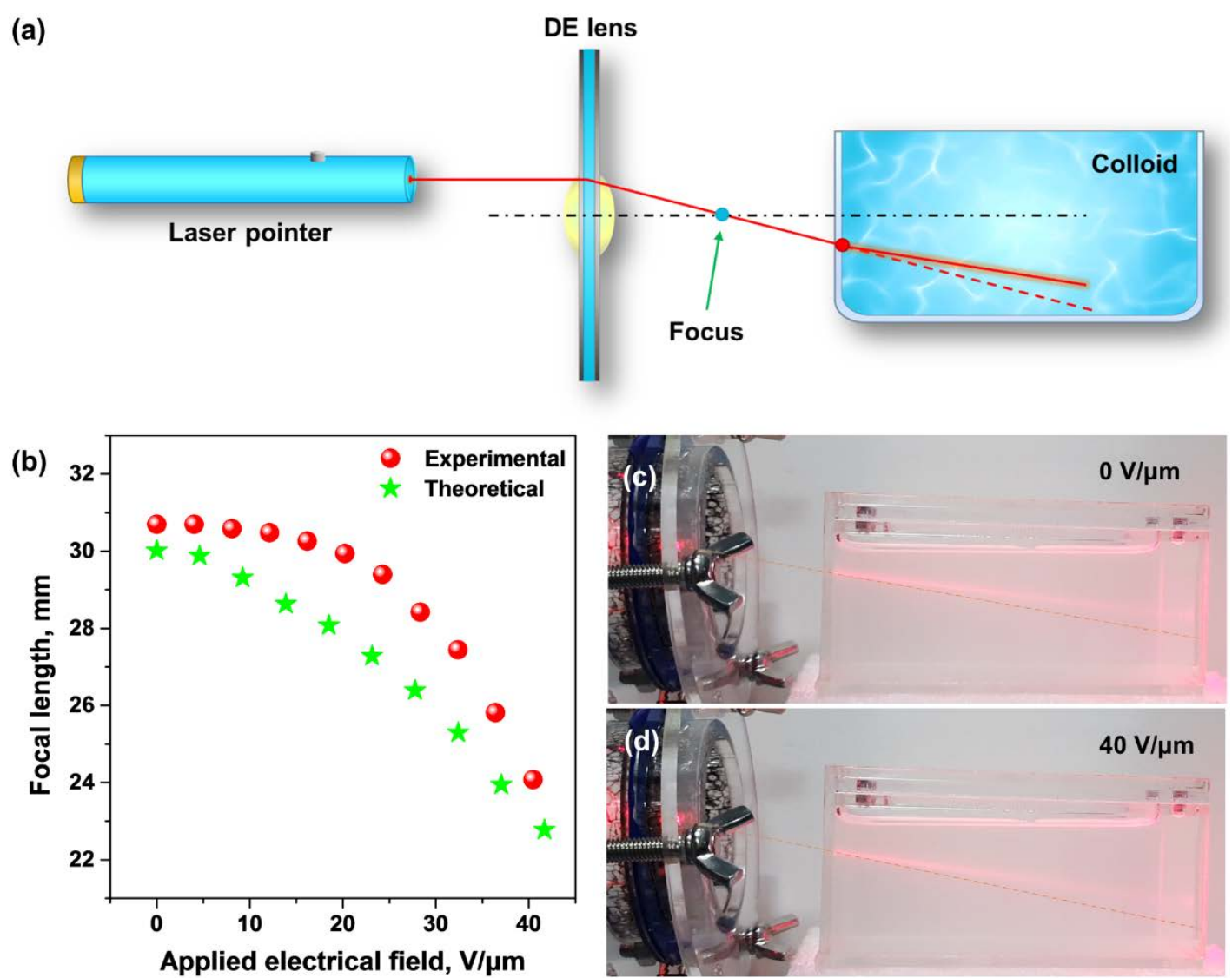

Figure 7 (a) A schematic of the measurement of tunable focal length. (b) The

plots show the changes in focal length of the tunable lens with varying applied electric

field. The photographs show the focus change when the laser beam passed through

soya milk based colloid (c) at $0 \mathrm{~V} / \mu \mathrm{m}$ and (d) at $40 \mathrm{~V} / \mu \mathrm{m}$.

Figure 7(a) showed a diagrammatic representation of the measurement of tunable focal length by observing the movement of a laser beam emitted from the lens. The whole electrical actuation process was presented in Video S4. When the electric field was applied, the DEA squeezed the lens to change its focus, then the direction of the converged laser beam was correspondingly changed. It can be seen from Figure 7(b) that the focal length decreased from $33.6 \mathrm{~mm}$ at $0 \mathrm{~V} / \mu \mathrm{m}$ to $26.1 \mathrm{~mm}$ at $40 \mathrm{~V} / \mu \mathrm{m}$. In this work, the surface of the lens could be approximated as two equal spherical caps for the evaluation of the change in focal length of the lens driven by DEAs [9]. The curvature 
radius $(R)$ and focal length $(f)$ of the lens can be obtained by employing the following geometric relationships:

$$
\begin{aligned}
& R=\frac{h^{2}+(2 r)^{2}}{2 h} \\
& \frac{1}{f}=\left(\frac{n_{l}}{n_{m}}-1\right)\left(\frac{1}{R_{1}}-\frac{1}{R_{2}}\right)
\end{aligned}
$$

where $h$ is the height of the caps, $r$ is the radius of the lens, $n_{l}$ is the refractive index of the lens and $n_{m}$ is the refractive index of the medium in which the lens is located. Because the lens is surrounded by air, the $n_{l} / n_{m}$ is about $1.43 . R_{1}$ and $R_{2}$ are the curvature radii of the two spherical caps of the lens, respectively. When $R_{1}$ is equal to $R_{2}$, Equation (5) can be simplified to Equation (6)

$$
\frac{1}{f}=\frac{2}{R}\left(\frac{n_{l}}{n_{m}}-1\right)
$$

During testing, the exact changes in $h$ (in the range of about $0.40 \mathrm{~mm}$ to $0.46 \mathrm{~mm}$ ) were measured by a laser displacement sensor and the real-time changes in $r$ in the range of $4.5 \mathrm{~mm}$ to $4.25 \mathrm{~mm}$ were recorded by a high-speed camera controlled by LabVIEW software. By substituting the values of $h$ and $r$ into Equation (4) and then into Equation (6), the focal lengths were calculated. As shown in Figure 7(b), the focal length changed from $30.1 \mathrm{~mm}$ to $22.8 \mathrm{~mm}$ as the electric field force increased from $0 \mathrm{~V} / \mu \mathrm{m}$ to $41 \mathrm{~V} / \mu \mathrm{m}$. It can be concluded that the experimental results were consistent with the simulation, giving a reliable indication of the lens deformation. 

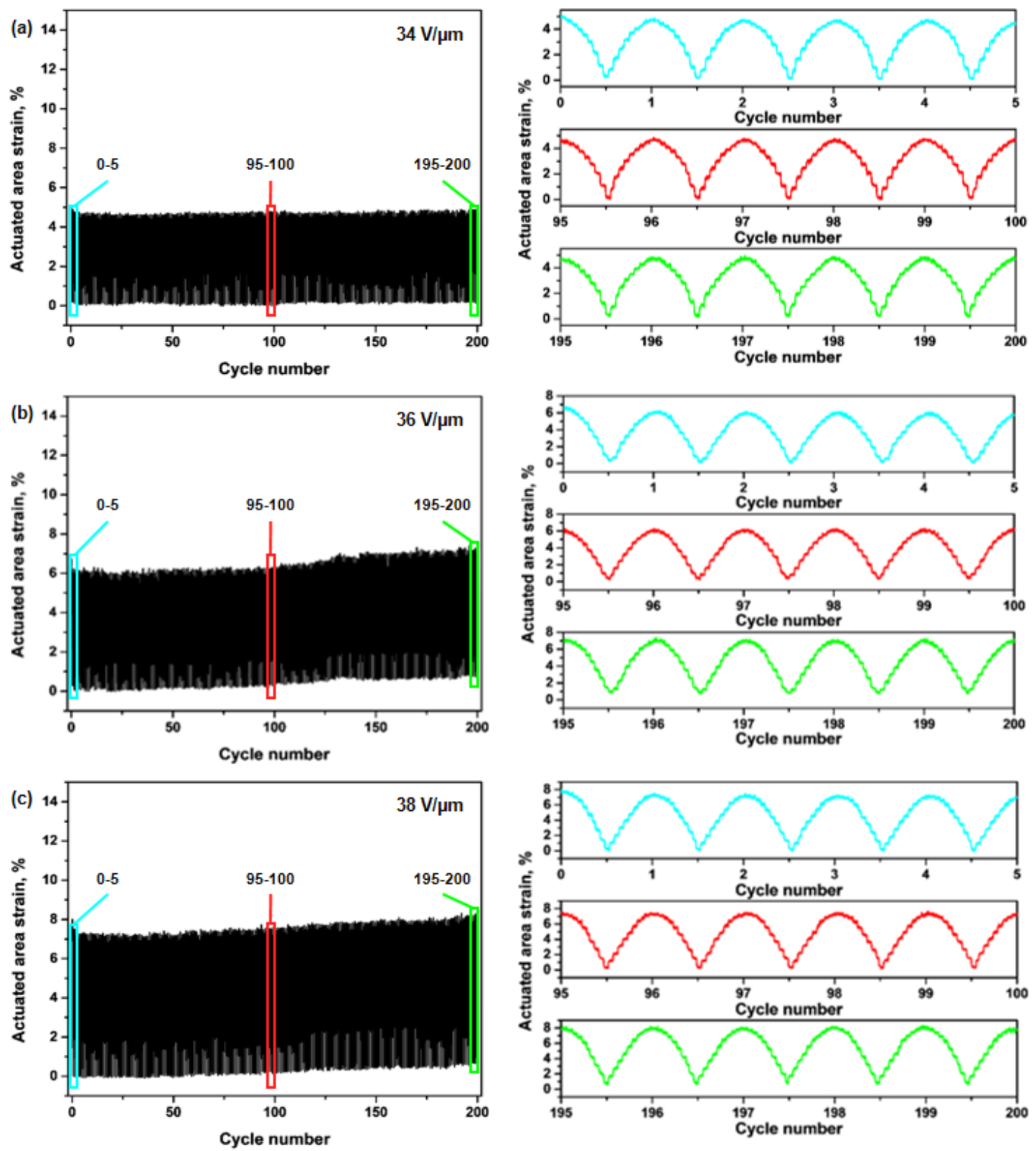

Figure 8 Continuously recorded changes in actuated area strain of a tunable lens

during 200 cycles of actuation tests under the applied electrical field from $0 \mathrm{~V} / \mu \mathrm{m}$ to $34 \mathrm{~V} / \mu \mathrm{m}, 36 \mathrm{~V} / \mu \mathrm{m}$ and $38 \mathrm{~V} / \mu \mathrm{m}$ respectively. Insets are the first five cycles (in blue) and the last five cycles (in green) of the reversible actuation tests.

Working stability is of great significance for the tunable lenses achieving practical application. In this work, 200 cycles of fully reversible loading were conducted under different maximum applied electrical field of $34 \mathrm{~V} / \mu \mathrm{m}, 36 \mathrm{~V} / \mu \mathrm{m}$ and $38 \mathrm{~V} / \mu \mathrm{m}$ with a 
stepped voltage of $200 \mathrm{~V}$ per $3 \mathrm{~s}$ from $0 \mathrm{~V}$. The variation of actuated strain of the tunable lens with cycles is illustrated in Figure 8. It can be seen that when the electrical field increased from 0 to $34 \mathrm{~V} / \mu \mathrm{m}$, the actuated strain increased by about $4.7 \%$ in the first five cycles and then about $4.9 \%$ in the last five cycles. When the electrical field increased from 0 to $36 \mathrm{~V} / \mu \mathrm{m}$, the maximum actuated area strain in the first five cycles (6.0\%) was $1.2 \%$ lower than that in the last five cycles (7.2\%), while the actuated area strain changed from $7.3 \mathrm{wt} \%$ in the first five cycles to $8.2 \%$ in the last five cycles when the electrical field increased from 0 to $38 \mathrm{~V} / \mu \mathrm{m}$. It can be seen that during 200 cycles of reversible actuation testing, the actuated area strains of DE lenses gradually became larger with the increasing number of test cycles and this was more dominant at higher applied electrical field. This is primarily due to the existence of the elastic hysteresis phenomenon in which a current deformation is affected by all previous deformations when the specimen is subjected to cyclic loading as in the case of this DE material [37]. Despite this, the DE lens performance exhibited good repeatability in 200 cycles of fully reversed loading. Furthermore, due to the limitation of the high voltage power source, the response speed of the tunable lens has not been characterized and will be done in the future work.

\section{Conclusions}

A polymer-based tunable lens has been developed combining a DE membrane actuator with a polymeric lens structure working as an aperture when the DE induces a curvature deformation. A relatively low ink viscosity to provide good fluidity was required to obtain a flat patterned film. However, this good fluidity of ink can lead to a deterioration 
in printing resolution. Based on this consideration, the pure SR and SR/3wt\% CuPc composite inks with a micro printing resolution were fabricated in this research. Though this offered a relatively low printing resolution, it still allowed the film to maintain a credible programmable shape. Also, the SR/3wt\% CuPc composite which has been employed to drive the lens prepared by EHD 3D printing possessed excellent electromechanical properties. The tunable lens had the ability of adjusting the focal length from $33.6 \mathrm{~mm}$ to $26.1 \mathrm{~mm}$ and exhibited a stable working life for 200 cycles. The defects in the interfaces between the lens encapsulated layer and the DE component might be generated during printing. This was regarded as the single reason for the inferior electromechanical properties in the experiments when compared with the simulation. However, it must be borne in mind that the simulation was merely a computer representation of the novel device and as such is limited by all of the limitations imposed by modelling. To sum up, this method has the potential to allow the design of 3D objects using virtually any composition with different structures and functionalities and gives an effective way of preparing inexpensive soft lenses by integrating DEs comprising transparent elastomer films filled with dielectric particles.

\section{CRediT authorship contribution statement}

Liang Jiang: Methodology, Investigation, Writing - original draft. Yuhao Wang: Investigation, Resources, Writing - original draft. Xueqin Wang: Investigation, Methodology. Fanggang Ning: Methodology, Validation. Shipeng Wen: Supervision, Validation. Yanfen Zhou: Conceptualization, Supervision, Writing - review \& editing, Funding acquisition. Shaojuan Chen: Conceptualization, Methodology, Supervision. 
Anthony Betts: Writing - review \& editing. Stephen Jerrams: Writing - review \& editing. Fenglei Zhou: Writing - review \& editing, Supervision.

\section{Acknowledgements}

The authors gratefully acknowledge the National Natural Science Foundation of China (Grant no. 51703108), the Shandong Provincial Natural Science Foundation, China (Grant no. ZR2017BEM042) and the Shandong Provincial Key Research and Development Program, China (Grant no. 2019GGX102071) and Shandong “Taishan Youth Scholar Program” for financial support.

\section{Supporting Information}

Video S1 showing the compliant electrode printing; Video S2 showing the experimental deformation of the tunable lens under an applied electrical field from $0 \mathrm{~V} / \mu \mathrm{m}$ to 41 V/ $\mu \mathrm{m}$; Video S3 showing the simulated deformation of the tunable lens under an applied electrical field from $0 \mathrm{~V} / \mu \mathrm{m}$ to $41 \mathrm{~V} / \mu \mathrm{m}$; Video S4 showing the movement of the laser beam under five cycles of applied electrical field from $0 \mathrm{~V} / \mu \mathrm{m}$ to $40 \mathrm{~V} / \mu \mathrm{m}$.

\section{References}

[1] Xiao Y, Mao J, Shan Y, Yang T, Chen Z, Zhou F, et al. Anisotropic Electroactive Elastomer for Highly Maneuverable Soft Robotics. Nanoscale. 2020:12:7514.

[2] Li J, Wang Y, Liu L, Xu S, Liu Y, Leng J, et al. A Biomimetic Soft Lens Controlled by Electrooculographic Signal. Advanced Functional Materials. 2019;29:1903762.

[3] Nguyen N. Micro-optofluidic Lenses: A review. Biomicrofluidics. 2010;4:031501.

[4] Pieroni M, Lagomarsini C, De Rossi D, Carpi F. Electrically tunable soft solid lens inspired by reptile and bird accommodation. Bioinspiration \& Biomimetics. 
2016;11:065003.

[5] Hwang T, Kwon H, Oh J, Hong J, Hong S, Lee Y, et al. Transparent actuator made with few layer graphene electrode and dielectric elastomer, for variable focus lens. Applied Physics Letters. 2013;103:023106.

[6] Krogmann F, Monch W, Zappe H. Electrowetting for Tunable Microoptics. IEEE/ASME Journal of Microelectromechanical Systems. 2008;17:1501-12.

[7] Ren H, Xianyu H, Xu S, Wu S. Adaptive dielectric liquid lens. Optics Express. 2008;16:14954-60.

[8] Carpi F, Frediani G, Turco S, Rossi DD. Bioinspired Tunable Lens with MuscleLike Electroactive Elastomers.21:4152-8.

[9] Maffli L, Rosset S, Ghilardi M, Carpi F, Shea H. Ultrafast All-Polymer Electrically Tunable Silicone Lenses. Advanced Functional Materials.2020:25:1656-65.

[10] She A, Zhang S, Shian S, Clarke DR, Capasso F. Adaptive metalenses with simultaneous electrical control of focal length, astigmatism, and shift. Science Advances. 2018;4.

[11] Yun S, Park S, Park BJ, Nam S, Park SK, Kyung K. A thin film active-lens with translational control for dynamically programmable optical zoom. Applied Physics Letters. 2015;107:081907.

[12] Zhang Y, Ellingford C, Zhang R, Roscow J, Hopkins MA, Keogh P, et al. Electrical and Mechanical Self-Healing in High-Performance Dielectric Elastomer Actuator Materials. Advanced Functional Materials. 2019;29:1808431.

[13] Duduta M, Hajiesmaili E, Zhao H, Wood RJ, Clarke DR. Realizing the potential 
of dielectric elastomer artificial muscles. Proceedings of the National Academy of Sciences of the United States of America. 2019;116:2476-81.

[14] Brochu P, Pei Q. Advances in Dielectric Elastomers for Actuators and Artificial Muscles. Macromolecular Rapid Communications. 2010;31:10-36.

[15] Petralia MT, Wood RJ. Fabrication and analysis of dielectric-elastomer minimumenergy structures for highly-deformable soft robotic systems. IEEE/RSJ International Conference on Intelligent Robots and Systems, Taipei, 2010. p. 2357-63.

[16] Wingert A, Lichter MD, Dubowsky S. On the design of large degree-of-freedom digital mechatronic devices based on bistable dielectric elastomer actuators. IEEE/ASME Transactions on Mechatronics. 2006;11:448-56.

[17] Xie S, Ramson PF, Graaf DD, Calius EP, Anderson IA. An adaptive control system for dielectric elastomers. IEEE international Conference on Industrial Technology, Hong Kong, 2005. p. 335-40.

[18] Dai ZH, Wang YL, Liu LQ, Liu XL, Tan PH, Xu ZP, Kuang J, Liu Q, Lou J, Zhang Z: Hierarchical graphene based films with dynamic self-stiffening for biomimetic artificial muscle. Advanced Functional Materials 2016; 26: 7003-7010.

[19] Plante J, Dubowsky S. On the performance mechanisms of Dielectric Elastomer Actuators. Sensors and Actuators A-physical. 2007;137:96-109.

[20] Nam JD, Choi HR, Koo JC, Lee YK, Kim KJ. Dielectric Elastomers for Artificial Muscles: Springer London; 2007.

[21] Keplinger C, Li T, Baumgartner R, Suo Z, Bauer S. Harnessing snap-through instability in soft dielectrics to achieve giant voltage-triggered deformation. Soft Matter. 
2012;8:285-8.

[22] Giousouf M, Kovacs G. Dielectric elastomer actuators used for pneumatic valve technology. Smart Materials and Structures. 2013;22:104010.

[23] Junshi Zhang, Hualing Chen. Voltage-induced beating vibration of a dielectric elastomer membrane, Nonlinear Dynamics. 2020;100:2225.

[24] Chen L, Busfield J J C, Carpi F. Electrically tunable directional light scattering from soft thin membranes. Optics Express, 2020:28:20669-14.

[25] Qiu Y, Zhang E, Plamthottam R, Pei Q. Dielectric Elastomer Artificial Muscle: Materials Innovations and Device Explorations. Accounts of Chemical Research. 2019;52:316-25.

[26] Yang D, Ni Y, Kong X, Wang Y, Zhang L. A mussel-like inspired modification of BaTiO3 nanopartciles using catechol/polyamine co-deposition and silane grafting for high-performance dielectric elastomer composites. Composites Part B: Engineering. 2019;172:621-7.

[27] Liashenko I, Rosell-Llompart J, Cabot A. Ultrafast 3D printing with submicrometer features using electrostatic jet deflection. Nature Communications. 2020;11:1-9.

[28] Liu X, Sun H, Liu S. Mechanical, dielectric and actuated properties of carboxyl grafted silicone elastomer composites containing epoxy-functionalized $\mathrm{TiO}_{2}$ filler. Chemical Engineering Journal. 2020:393:124791.

[29] Onses MS, Sutanto E, Ferreira PM, Alleyne AG, Rogers JA. Mechanisms, Capabilities, and Applications of High-Resolution Electrohydrodynamic Jet Printing. 
Small. 2015;11:4237-66.

[30] Zhang G, Lan H, Qian L, Zhao J, Wang F. A Microscale 3D Printing Based on the Electric-Field-Driven Jet. 3D Printing and Additive Manufacturing. 2020:0:1.

[31] Wang D, Zhao X, Lin Y, Liang J, Ren T, Liu Z, et al. Nanoscale coaxial focused electrohydrodynamic jet printing. Nanoscale.10.1039.C8NR01001C.

[32] Li JL. EHD sprayings induced by the pulsed voltage superimposed to a bias voltage. Journal of Electrostatics.2007:65:750-7.

[33] Kim S-Y, Kim Y, Park J, Hwang J. Design and evaluation of single nozzle with a non-conductive tip for reducing applied voltage and pattern width in electrohydrodynamic jet printing (EHDP). Journal of Micromechanics and Microengineering. 2010;20:055009.

[34] Hashimdeen SH. A prototype for 3D electrohydrodynamic printing: UCL (University College London); 2016.

[35] Lee H, Seong B, Kim J, Jang Y, Byun D. Direct Alignment and Patterning of Silver Nanowires by Electrohydrodynamic Jet Printing. Small. 2014;10:3918-22.

[36] Wang Y, Zhou Y, Li W, Liu Z, Zhou B, Wen S, et al. The 3D printing of dielectric elastomer films assisted by electrostatic force. Smart Materials and Structures. 2020;30(2):025001.

[37] Jiang L, Wang Y, Wen S, Zhou Y, Ma J, Chen S, et al. Fabrication of dielectric elastomers with improved electromechanical properties using silicone rubber and walnut polyphenols modified dielectric particles. Materials \& Design. 2020;192:108674. 